\title{
Reduce the Human Antibiotic Resistances: Smart-WC and Good Practices?
}

\author{
Giancarlo Ranalli* \\ Department of Bioscience and Territory, University of Molise, Italy \\ *Corresponding author: Giancarlo Ranalli, Department of Bioscience and Territory, University of Molise, Italy. \\ To Cite This Article: Giancarlo Ranalli, Reduce the Human Antibiotic Resistances: Smart-WC and Good Practices?. Am J Biomed Sci \& Res. 2019 - \\ 5(1). AJBSR.MS.ID.000877. DOI: 10.34297/AJBSR.2019.05.000877
}

Received: 眥 September 05, 2019; Published: 眥 September 09, 2019

Keywords: Antibiotic resistances; Smart-WC; Good practices

\section{Introduction}

The use and abuse of antibiotics in several systems for human and animal therapy, in industrial and agricultural application have induced into the spead in different environmental habitats an accumulation of high level/concentration of such agents. These compounds caused alteration of the natural microbial balance by the suppressiveness of great number of positive non-pathogenic bacteria and the increase in the amount of negative drug resistant pathogenic bacteria. Antibiotic resistance is the ability of bacteria to be or become resistant to antibiotics, thus managing to survive and multiply even in the presence of the drug. This is a public health problem at European and global level, due to the excessive and improper use of these drugs. The alarm is recurrent! In 2012, the World Health Organization (WHO) and the European Center for Prevention and Control of Diseases reported that there was an increase in Europe of resistance in Gram negative pathogens such as Escherichia coli, Klebsiella pneumoniae, Pseudomonas aeruginosa. The data of the most recent WHO report 2017 and of the European Center for the Control of Infectious Diseases (ECDC) state that due to the resistance of bacteria to antibiotics there are over 671,000 cases of infection, to which 33,000 deaths are attributable.

The constant increase in the environment of antibioticresistant microbial forms is real emergency and therefore every year results in a significant increase in deaths caused by microbial infection from resistant strains. Antibiotic resistance is a European (or better global) phenomenon that has been constantly spreading in recent decades. The introduction of penicillin in 1929 offered the possibility of eradicating infectious diseases but also caused the appearance of bacteria resistant to the action of the antibiotics themselves. In fact, a large part of the drugs we take is eliminated as such by urine and faeces, while the existing purifiers are designed to reduce the organic load in the wastewater but not to eliminate the drugs that may be present and very diluted in them. In fact, these plants work through biological systems of activated sludge or rotating disks and are based on the presence of a microbial community adapted to decompose the easily biodegradable organic substances of which they feed, thus impoverishing the discharge. However, the microflora in sewage treatment plants is not able to eliminate the cocktail of antibiotics (non biodegradable) which are therefore found into the aquatic environment as sea, rivers and lakes causing bio-accumulation $[1,2]$.

To date, antibiotics and metabolites in wastewater would not have a direct negative effect on people but on environmental bacteria, increasing their resistance to antibiotics: an example among the many is Escherichia coli. Releasing of antibiotics by human beings and animals depends mainly on the consumption rates of antibiotics: Spain, Italy, Portugal and Greece are countries that consume more antibiotics than other Northern European countries [3]. Since 2012, very little or nothing has been done. We must act and immediately to stop the risk to which humanity is exposed. Today the solutions can only partly derive from already known considerations (total separation between classes of antibiotics for zootechnical use and for human use; more information on the good use of antibiotics and on the risks of abuse; compliance with therapies in cases of administration, avoid misuse and do it yourself, etc.). But all this is not enough; if we really want to interrupt the cycle that lasts between use (although correct as prescribed by correct diagnosis of the disease) and release of antibiotics into the environment, we must start from the awareness of having to intervene from different perspectives as well as from concrete and innovative actions: the devices Smart-WCs type can be a valuable contribution.

\section{Comments}

An Italian interdisciplinary research group [4-6] composed of chemists, microbiologists, pharmacologists, statisticians, has 
in study and experimentation a Smart-WC prototype equipped with a device that can guarantee, through chemical catalysts, the elimination / destruction / deactivation of residues of antibiotics and metabolites, and associate microflora potentially carrying resistance. The new "original" proposal is to develop a new "homecleaning antibiotics technology" vs antibiotic resistance, based on the domestic treatment of antibiotic residues: it is in the bathroom that the individual is subjected to antibiotic therapy for a limited time, generally a few days, faeces and urine are released / containing a portion of antibiotic residues and metabolites (clearance). It is here, in bathroom, that action must be taken immediately, as the time zero, therefore "upstream" and not "downstream" of the wastewater treatment plants. The proposed technological solution allows the proportion of waste actually containing antibiotic residues in humans to be treated "only" in a targeted manner during those days of therapy; it is therefore an action in a reduced space, volume and time. This means into high degradation efficiency, as well as cost reduction.

The following are avoided:

a) Effects of dilutions between few wastewaters with antibiotics and large volumes of wastewater without antibiotics;

b) The establishment of drug-resistance gene transfer processes in the microflora in sewer pipes,

c) The difficulties to make changes to the wastewater treatment plants already in use. The new technology defined Smart-WC provides a device both for existing wc and for new homes, able to reduce the presence of antibiotics in faeces and urine just produced by subjects in antibiotic therapy. In practice, these are toilets equipped with a system to dispense chemical reagents (eg tablets) containing nano titanium dioxide particles or Fenton reagents, directly in the toilet bowl, readily able to initiate drug degradation, thus avoiding induction over time of the resistance of the bacteria to antibiotics. In the case of nanoparticles, these can be used with absorbent materials with a high surface area (recovery by sedimentation) or with magnetic materials, which are themselves recovered downstream in industrial sewage sludge and thus make the process environmentally friendly. The problem of antibiotic resistance is complex, however important contributions can also arise from suitable governance choices of the antibioticresistance problem. In fact, among the good practices that can be adopted in urban environments, with a high level of population density, with services dedicated to transport, commerce, tourism (eg metro, airports, train stations, highways stations - motorway services, cinema, theater, etc.) an additional contribution may derive from dedicated toilets for people who are at the end of their antibiotic-based therapeutic treatment: for them, a dedicated Smart-WC -distinguishable, lile. yellow in color, compared to the common white ones. The "war" against antibiotic resistance will result in a true victory only if adequate and targeted campaigns of correct information are adopted for citizens in all coutries: new sustainability solutions combined to ad hoc environmental policies.

\section{References}

1. Kümmerer K (2009) Antibiotics in the aquatic environment-a reviewPart I. Chemosphere 75: 417-434.

2. Alaton I, Dogruel S (2004) Pre-treatment of penicillin formulation effluent by advanced oxidation processes. J Hazard Mater 112: 105-113.

3. Ayse K, Berna KM, Nihan Ö, Özge S, Taner Y, et al. (2017) Treatment of antibiotics in wastewater using advanced oxidation processes (AOPs).

4. Bressan M, Liberatore L, D’Alessandro N, Tonucci L, Belli C, et al. (2004) Improved combined chemical and biological treatment on olive oil mill wastewaters. J Agricult Food Chem 52: 1228-1233.

5. Liberatore L, Bressan M, Belli C, Lustrato G, Ranalli G (2012) Chemical and biological combined treatments for the removal of pesticides from wastewaters. Water Air Soil Pollution 223(8): 4751-4759.

6. Rampazzi L, Andreotti A, Bressan M, Colombini MP, Corti C, et al. (2018) An interdisciplinary approach to a knowledge-based restoration: The dark alteration on Matera Cathedral (Italy). Applied Surface Science 458: 529-539. 